\title{
On Approximation of Real-World Influence Spread
}

Yu Yang, Enhong Chen, Qi Liu, Biao Xiang, Tong Xu, and Shafqat Ali Shad

Dept. of Computer Science, Univ. of Science and Technology of China, Hefei 230026, China

\{ryanyang, feiniaol, bxiang, tongxu, shafqat\}@mail.ustc.edu.cn,

cheneh@ustc.edu.cn

\begin{abstract}
To find the most influential nodes for viral marketing, several models have been proposed to describe the influence propagation process. Among them, the Independent Cascade (IC) Model is most widelystudied. However, under IC model, computing influence spread (i.e., the expected number of nodes that will be influenced) for each given seed set has been proved to be \#P-hard. To that end, in this paper, we propose $G S$ algorithm for quick approximation of influence spread by solving a linear system, based on the fact that propagation probabilities in real-world social networks are usually quite small. Furthermore, for better approximation, we study the structural defect problem existing in networks, and correspondingly, propose enhanced algorithms, GSbyStep and SSSbyStep, by incorporating the Maximum Influence Path heuristic. Our algorithms are evaluated by extensive experiments on four social networks. Experimental results show that our algorithms can get better approximations to the IC model than the state-of-the-arts.
\end{abstract}

\section{Introduction}

Recently, viral marketing has drawn more and more attention from both industrial and research fields [15]. This kind of marketing is based on the word-ofmouth effect in social networks, i.e., one may be influenced by his neighbors. According to a survey [11], $83 \%$ of people prefer consulting family, friends or an expert over traditional advertising before trying a new restaurant, $71 \%$ of people do the same before buying a prescription drug, and so on. Thus, companies believe that viral marketing should be one of the most effective marketing strategy.

Unlike traditional marketing strategies, viral marketing only targets a few influential individuals in a social network. For example, if a company employs a viral marketing strategy to promote sales performance, it only needs to choose a small number of individuals and persuade them to be the initial users(by offering them discounted or free products etc.). Due to the word-of-mouth effect, these initial users, also called seed users, will influence their friends to use this product. And once the friends are influenced, they will try to influence their friends and so on. Eventually, a much larger group of people compared to the number of initial users will adopt this product. 
Since different seed users usually lead to different results of word-of-mouth propagation, an important step for a successful viral marketing is to precisely evaluate the expected number of influenced individuals (influence spread) for each seed user set. Along this line, several influence models have been proposed [8][9][12]. Among these models, Independent Cascade(IC) Model is a simple and most widely used one that describes the information propagation in a social network as a stochastic process according to certain probabilistic rules [9][12]. However, Wei Chen et al. have proved that computing influence spread of a set of seed users, under IC model, is a \#P-hard problem [7]. As an alternative, Monte Carlo simulation, which is very time-consuming, is employed to approximately calculate influence spread. For example, for a moderate sized social network, we usually have to run Monte Carlo simulation for more than ten thousands times to obtain a good estimation of the true influence spread. Thus, the problem of efficiently computing influence spread has become a bottleneck for the deployment of viral marketing.

To that end, in this paper, we provide a novel study on approximating influence spread under IC model, mainly based on the observation that the influence propagation probabilities in real-world social networks are usually quite small. Specifically, our main contributions can be summarized as follows:

- To address the above efficiency problem, we show that by solving a linear system we can approximately calculate each node (individual, or user)'s probability of being influenced, and thus we can quickly compute influence spread of a given seed set.

- We point out that SteadyStateSpread algorithm [1] is also an approximation for the real influence spread under IC model when the propagation probabilities are small, and this was not illustrated by Aggarwal, et al. in [1].

- For better approximation, we discover the structural defect problem (as illustrated by Definition 2 in Section 4.1) existing in networks, and further propose enhanced algorithms by incorporating the Maximum Influence Path(MIP) heuristic [7] to both our algorithm and SteadyStateSpread.

- We evaluate our algorithms on four real networks. Experimental results prove our discoveries and show that the proposed algorithms can get good approximations of the real influence spread. Moreover, the nodes ranking obtained by our algorithms are very similar to the true ranking results.

\section{Related Work}

In general, related work can be grouped into two categories. The first category includes the most relevant work on influence models. In the second category, we introduce the related work on computing social influence spread.

Influence Models. Domingos et al. first proposed to mine social networks for marketing [8]. However, their models are essentially descriptive, and prior works $[1][7][12][13]$ following [8] focus more on the models that explicitly represent the step-by-step dynamics of influence. 
Among existing influence models, Independent Cascade(IC) Model [9][12] is one of the most widely-studied models. In IC model, a social network is represented by a directed graph $G(V, E)$. Each node $v \in V$ denotes an individual in the social network. Each edge $(u, v)$ represents the relationship between $u$ and $v$, and is assigned with a real number $p_{u v}\left(p_{u v} \in[0,1]\right)$ which is the probability that $v$ is influenced by $u$ through the edge in the next step after $u$ is activated. In the beginning, there is a set of users (seed set) who are already activated, i.e. influenced, denoted by $S$. Let $S_{t}$ represents the set of nodes that become activated at time $t$, and $S_{0}=S$. At time $t+1$, each node $u \in S_{t}$ will try to activate its inactivated neighbor $v$ with probability $p_{u v}$. If there are no newly activated nodes at time $t+1$, the propagation process ends. The influence spread of $S$, which is denoted by $\sigma(S)$, is the expected number of nodes being activated in the whole propagation process.

Two kinds of IC model, namely Uniform IC Model and Weighted Cascade(WC) Model are defined in [12]. In uniform IC model, each link shares the same propagation probabilities, while according to WC model, the propagation probability through edge $(u, v)$ equals to weight $(u, v) /$ indegree $(v)$.

Influence Spread Computation. In [12], Kempe, et al. exploited running Monte Carlo simulation for a large number of times to evaluate the influence spread of a given seed set under IC model.

Further, Wei Chen et al. [7] proved that computing influence spread based on IC model is \#P-hard. Given the fact that the influence of a node is always local, they proposed the Maximum Influence Path(MIP) heuristic. Specifically, they defined the propagation probability of a path $P=<u=n_{1}, n_{2}, \ldots, n_{m}=$ $v>(u \in S), p p(P)$, as

$$
p p(P)=\prod_{i=1}^{m-1} p_{n_{i} n_{i+1}}
$$

and the maximum influence path from $S$ to $v$, which is denoted by $\operatorname{MIP}(S, v)$, is defined as

$$
M I P(S, v)=\arg \max _{P}\{p p(P) \mid P \in \operatorname{Path}(S, v)\}
$$

in which $\operatorname{Path}(S, v)$ is the set of all paths from $S$ to $v$. By using the MIP heuristic, they regard the probability of $v$ being influenced by seed set $S$ as $p p(M I P(S, v))$. Their experiments showed that by using this heuristic one can accelerate the influence maximization algorithm drastically. However, the influence spread calculated by the MIP heuristic maybe very different with the true influence spread because it abandons too many possible paths.

In contrast, Kimura and Saito proposed the shortest-path based influence models and provided efficient algorithms to compute influence spread under these models in [13]. However, their algorithms are only suitable for the uniform IC model where propagation probabilities of links are all the same, which is seldom the case in reality.

Recently, Aggarwal et al. [1] proposed the SteadyStateSpread method to compute flow authority by solving a system of nonlinear equations, 


$$
p_{v}= \begin{cases}1 & \text { if } v \in S \\ 1-\prod_{u \in N(v)}\left(1-p_{u v} * p_{u}\right) & \text { if } v \notin S\end{cases}
$$

in which $N(v)$ is the set of all $v$ 's in-neighbors and $p_{v}$ indicates the probability of $v$ being influenced. As we will explain later, Eq.(1) can be used to approximately compute the real influence spread under IC model when propagation probabilities through links are small. However, it does not strictly hold for some situations(e.g., the situation illustrated in Section 4.1). More importantly, there are some difficulties in solving systems of nonlinear equations, such as the convergence problem [6] and the multiple solutions problem [10]. Though Aggarwal et al. [1] gave a simple iterative algorithm for solving Eq.(1), they did not theoretically prove that their algorithms can converge or Eq.(1) has only one solution.

\section{Approximating Influence Spread by Linear System}

In this section, we first illustrate the observation that influence propagation probabilities in real-world social networks are usually quite small. Based on this fact, we then show the way to approximate influence spread, and represent the approximation by a linear system. At last, we propose a simple iterative algorithm to solve the linear system and return the influence spread for each seed set.

\subsection{Preliminary Observation}

Though there exists the effect of peer-to-peer influence, in real scenarios of information diffusion, propagation probabilities between people are very small. For example, according to [5], in Facebook, the average probability that an individual will share a Web link is about $2 \%$ when there are 6 of his friends who shared the same link before. In LiveJournal, the average probability that an individual will join a community is no more than $2 \%$ even though 50 of his/her friends have already joined that community [4]. Moreover, influence is not the only reason that leads to phenomena of friends sharing same Web links and joining same communities. Prior works, [2][3][16], showed that correlations between friends in social networks are also an important factor that contributes to homophily phenomena. Thus, the actual propagation probability caused by the effect of influence is even smaller.

To facilitate the following discussion, we first define small propagation probabilities mathematically.

Definition 1 (Small Propagation Probabilities). Given a network $G=$ $(V, E)$, if for $\forall v \in V$, and for $\forall u \in N(v), p_{u v}<\operatorname{weight}(u, v) /$ indegree $(v)$, we say the propagation probabilities in $G$ are small. Note that indegree $(v)=$ $\sum_{u \in N(v)}$ weight $(u, v)$.

From Definition 1 we can see that if the small propagation probabilities condition holds in a social network, then for $\forall v \in V, \sum_{u \in N(v)} p_{u v}<1$ because $p_{u v}<$ weight $(u, v) /$ indegree $(v)$. The further explanations of these two formulas can be 
easily observed from the real world social networks: First, besides the influence coming from the direct neighbors in the specific social network, each node $v$ 's activity is also impacted by the information from other sources (e.g., influence coming from other friends that are not included in the current network). Second, when influence propagates in the network, there is information lost (or decay) in each node, and this has been widely observed and adopted by models from other domains, such as the PageRank method [14].

\subsection{Approximation of Real-World Influence Probability}

In this subsection we consider approximating influence spread under IC model for social networks, where propagation probabilities are small. We use $\sigma(S)$ to denote influence spread of a seed set $S$. It is obvious that $\sigma(S)=\sum_{v} p_{v}$, in which $p_{v}$ denotes the probability that $v$ will be influenced, also called the influence probability of $v$. Since $\forall v \in S, p_{v}=1$, the core step of computing $\sigma(S)$ is to compute $p_{v}$ for each $v \notin S$. In the following, we show that the influence probability of $v(v \notin S)$ can be well approximated by a linear equation that $p_{v}=\sum_{u \in N(v)} p_{u} * p_{u v}$.

Let $v(t)$ denotes the event that $v$ becomes influenced at time $t$. Note that $p\{v(0)\}=1$ if $v \in S$. For each node $v \notin S, p\{v(t)\}$ can be computed by

$$
p\{v(t)\}=\sum_{W \subseteq N(v)} p\{v(t) \mid W(t-1), \tilde{v}(t-1)\} p\{W(t-1), \tilde{v}(t-1)\}
$$

where $N(v)$ is the set of in-neighbors of $v, W$ is a subset of $N(v), W(t-1)$ indicates the event that all nodes in set $W$ become influenced at time $t-1$ and $\tilde{v}(t-1)$ denotes the event that $v$ is still not influenced after time $t-1$. Worth noting that $p\{v(t)\}=p\{v(t), \tilde{v}(t-1)\}$ 1. It is obvious that

$$
p\{v(t) \mid W(t-1), \tilde{v}(t-1)\}=1-\prod_{u \in W}\left(1-p_{u v}\right)
$$

Following [8] and [13], given the marginals $\{p\{u(t-1)\} ; u \in W\}$ and $p\{\tilde{v}(t-1)\}$, we approximate $p\{W(t-1), \tilde{v}(t-1)\}$ by the maximal entropy estimation:

$$
p\{W(t-1), \tilde{v}(t-1)\}=p\{\tilde{v}(t-1)\} \prod_{u \in N(v)} p\{u(t-1)\}^{h_{u}}(1-p\{u(t-1)\})^{1-h_{u}}
$$

where $h_{u}$ is an indicator that if $u \in W, h_{u}=1$, otherwise $h_{u}=0$. Combining Eq.(2), Eq.(3) and Eq.(4), and after some algebraic transformations, we have

$$
p\{v(t)\}=p\{\tilde{v}(t-1)\}\left[1-\prod_{u \in N(v)}\left(1-p_{u v} * p\{u(t-1)\}\right)\right]
$$

Note that under the small propagation probabilities condition, $p_{u v}$ is very small, the probability that $v$ will be influenced is even smaller. So $p\{\tilde{v}(t-1)\}$ is very

\footnotetext{
${ }^{1}$ If $v$ is influenced at time $t$, it should stay inactive from time 0 to $t-1$.
} 
close to 12 . Thus, $p\{v(t)\}$ can be approximated by

$$
p\{v(t)\}=1-\prod_{u \in N(v)}\left(1-p_{u v} * p\{u(t-1)\}\right)
$$

Since $p_{u v} * p\{u(t-1)\}<<1$ and $(1-a)(1-b) \approx 1-a-b$ when $a, b<<1$, we can further approximate $p\{v(t)\}$ with a linear equation:

$$
p\{v(t)\}=\sum_{u \in N(v)} p_{u v} * p\{u(t-1)\}
$$

Now we add up $p\{v(t)\}$ over $t$ to get $p_{v}$. Note that $p_{v}=1$ if $v \in S$. For $v \notin S$, we have

$$
\begin{aligned}
p_{v} & =\sum_{t=0} p\{v(t)\} \\
& =p\{v(0)\}+\sum_{u \in N(v)} p_{u v} \sum_{t=1} p\{u(t-1)\} \\
& =\sum_{u \in N(v)} p_{u v} p_{u}
\end{aligned}
$$

Because we are discussing approximation under the condition that $\sum_{u \in N(v)} p_{u v}$ $<1$, it is guaranteed that $0 \leq p_{v}<1$. Thus, the influence probability $p_{v}$ computed by Eq.(8) is well defined.

Approximation From SteadyStateSpread. As have said, under the small propagation probabilities condition $p_{u v} * p_{u}<<1$, and recall Eq.(1), it is easy to conclude that $\sum_{u \in N(v)} p_{u v} p_{u} \approx 1-\prod_{u \in N(v)}\left(1-p_{u v} p_{u}\right)$. In this situation, we can see that SteadyStateSpread is actually also an approximation for the true influence spread. In the following experiment section, our experimental results verify that the smaller $p_{u v}$ is, the more accurate approximation will be obtained by SteadyStateSpread.

\subsection{Linear System Formulation}

Rewriting Eq.(8) in a matrix form we can get a linear system. To facilitate the following discussion, first we list some math notations in Table 1.

Table 1. Math Notations

\begin{tabular}{|l|l|}
\hline Notations & DESCRIPTION \\
\hline$[\mathbf{M}]_{|V| *|V|}$ & probability adjacent matrix, $\mathbf{M}_{u v}=p_{u v}$ \\
\hline $\mathbf{P}=\left[p_{1}, p_{2}, \ldots, p_{|V|}\right]^{T}$ & $p_{v}$ is the probability of $v$ being influenced by seed set $S$ \\
\hline $\mathbf{B}=\left[b_{1}, b_{2}, \ldots, b_{|V|}\right]^{T}$ & $b_{v}=1$ if $v \in S$, otherwise $b_{v}=0$ \\
\hline $\mathbf{M}_{\bar{S}}$ & $\begin{array}{l}\text { matrix which is cut down from matrix } \mathbf{M} \text { by removing rows } \\
\text { whose index } v \in S\end{array}$ \\
\hline $\mathbf{M}_{\overline{S S}}$ & $\begin{array}{l}\text { matrix which is cut down from matrix } \mathbf{M} \text { by removing rows and } \\
\text { columns whose index } v \in S\end{array}$ \\
\hline
\end{tabular}

${ }^{2}$ The assumption on this value will be verified in the experimental section 5.3. 
For node $v \notin S$, we rewrite Eq.(8) by

$$
p_{v}=\sum_{u \in N(v) \wedge u \notin S} p_{u v} * p_{u}+\sum_{u \in N(v) \wedge u \in S} p_{u v}
$$

Further, using matrix form to represent the right side of Eq.(9), we have

$$
p_{v}=\left(\mathbf{M}_{\bar{S}}\right)_{v}^{T} \mathbf{P}_{\bar{S}}+\left(\mathbf{M}^{T} \mathbf{B}\right)_{v}
$$

Put all nodes $v$ that $v \notin S$ together,

$$
\mathbf{P}_{\bar{S}}=\left(\mathbf{M}_{\overline{S S}}\right)^{T} \mathbf{P}_{\bar{S}}+\left(\mathbf{M}^{T} \mathbf{B}\right)_{\bar{S}}
$$

thus we have

$$
\left[\mathbf{I}-\left(\mathbf{M}_{\overline{S S}}\right)^{T}\right] \mathbf{P}_{\bar{S}}=\left(\mathbf{M}^{T} \mathbf{B}\right)_{\bar{S}}
$$

Given that we are dealing with approximations of influence spread under the condition that $\sum_{u \in N(v)} p_{u v}<1$, the matrix $\left[\mathbf{I}-\left(\mathbf{M}_{\overline{S S}}\right)^{T}\right]$ is strictly diagonally dominant. Thus, $\left[\mathbf{I}-(\mathbf{M} \overline{S S})^{T}\right]$ is invertible and the linear system of Eq.(12) has only one solution that $\mathbf{P}_{\bar{S}}=\left[\mathbf{I}-\left(\mathbf{M}_{\overline{S S}}\right)^{T}\right]^{-1}\left(\mathbf{M}^{T} \mathbf{B}\right)_{\bar{S}}$. In this way, by summing up $p_{v}$ over each $v$, we get influence spread for seed set $S($ i.e., $\sigma(S))$.

\subsection{A Simple Iterative Algorithm}

As we stated above, to approximately compute the influence spread of a seed set $S$, we only have to solve the linear equation Eq.(12) and sum up all $p_{v}$.

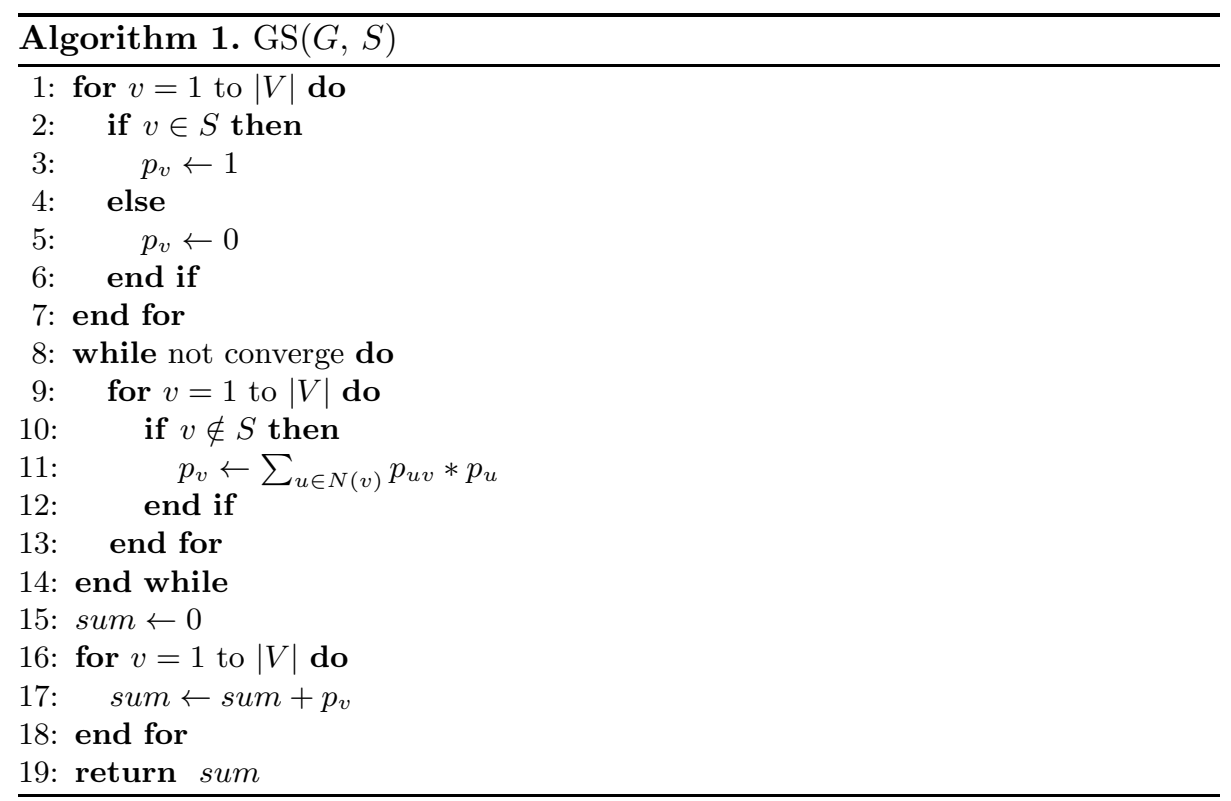


Since $\left[\mathbf{I}-\left(\mathbf{M}_{\overline{S S}}\right)^{T}\right]$ is strictly diagonally dominant, we can use Gauss-Seidel(GS) algorithm to efficiently solve Eq.(12) in $O(E)$ time. The entire computation process can be illustrated by Algorithm 1, where the iteration formula used is Eq.(8). Based on the output of Algorithm 1, we can rank each seed set $S$, so as to find the most influential individuals for marketing.

\section{Algorithms Incorporating MIP Heuristic}

In this section, we first illustrate the structural defect problem existing in many methods for social networks. Then, to address this problem and to get a better approximation, we propose enhanced algorithms by incorporating the Maximum Influence Path(MIP) heuristic into both $G S$ algorithm and SteadyStateSpread.

\subsection{Structural Defect}

According to Eq.(1) and Eq.(8), for $v \notin S$, the value of $p_{v}$ depends on all of its in-neighbors. However, in fact sometimes a node $u$ 's influence probability $p_{u}$ is independent from some of its in-neighbors. Consider the following example of an undirected network, as shown in Fig.1.

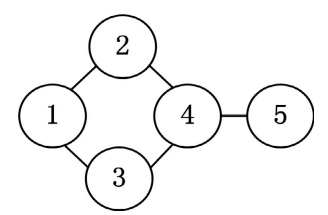

Fig. 1. An undirected network

In this undirected network, assume that node 1 is the only seed node. Based on Eq.(1) and Eq.(8), the probability of node 4 being influenced depends on the probability of node 5 being influenced. However, actually, to influence node 5 , node 4 should be influenced first. Thus, $p_{4}$ is irrelevant with $p_{5}$, and this contradicts with Eq.(1) and Eq.(8). We have reasons to believe that each network, especially an undirected network, may have many sub-structures like Fig.1, and in this situation, if we use Eq.(1) or Eq.(8) to calculate $p_{v}$ would get misleading results. In summary, we call structures like (node 4, node 5) in Fig.1 structural defect of Eq.(1) and Eq.(8), and we define it in a mathematical way.

Definition 2 (Structural Defect). Given a network $G=(V, E)$, a seed set $S$ and an influence spread algorithm $A$, if $\exists u, v \notin S, \forall P=\left(p_{n_{1}}, p_{n_{2}}, \ldots, p_{n_{m}}=v\right) \in$ $\operatorname{Path}(S, v), u \in P$ (that is, every path from $S$ to $v$ has to pass $u$ ), and according to $A$, the value of $p_{u}$ depends on $p_{v}$, we say that $(u, v)$ is a structural defect of algorithm $A$ on $G$. 


\subsection{Incorporating Maximum Influence Path Heuristic}

To handle the problem of structural defect, we propose to incorporate the Maximum Influence Path(MIP) heuristic [7] into our algorithm. The main idea is to set an iteration threshold $\beta(v)$ for node $v$, i.e., for each $v$, we only update $p_{v}$ in the first $\beta(v)$ iterations.

Let's consider the algorithms of Gauss-Seidel(GS) and SteadyStateSpread [1], which are both iterative processes of accumulating each path's influence probability for a node (e.g., $v$ ) by Eq.(8) and Eq.(1) respectively. Specifically, for a path $P$ from $S$ to $v$, if we want to include the propagation probability through $P$, we have to update $p_{v}$ for no less than length $(P)$ (hops of $P$ ) iterations. Since the maximum influence path is the path with biggest propagation probability, we do not want to abandon this path's influence. Thus, let step $[v]$ denotes hops of this maximum influence path from $S$ to $v$, and to include the influence probability of maximum influence path, we should set the iteration threshold for $v$ to be at least $s t e p[v]$.

However, since step $[v]$ is usually also the shortest distance from $S$ to $v$, and if we just update $p_{v}$ in the first $s t e p[v]$ rounds of iterations, we may miss some important influences from other paths. For example, the value of $p_{2}$ for node 2 in Fig. 1 does depend on the influence from all its neighbors. In contrast, if we update $p_{v}$ for too many iterations, the structural defect will have serious impact on the estimation of influence spread. Take node 4 in Fig.1 for example, as the

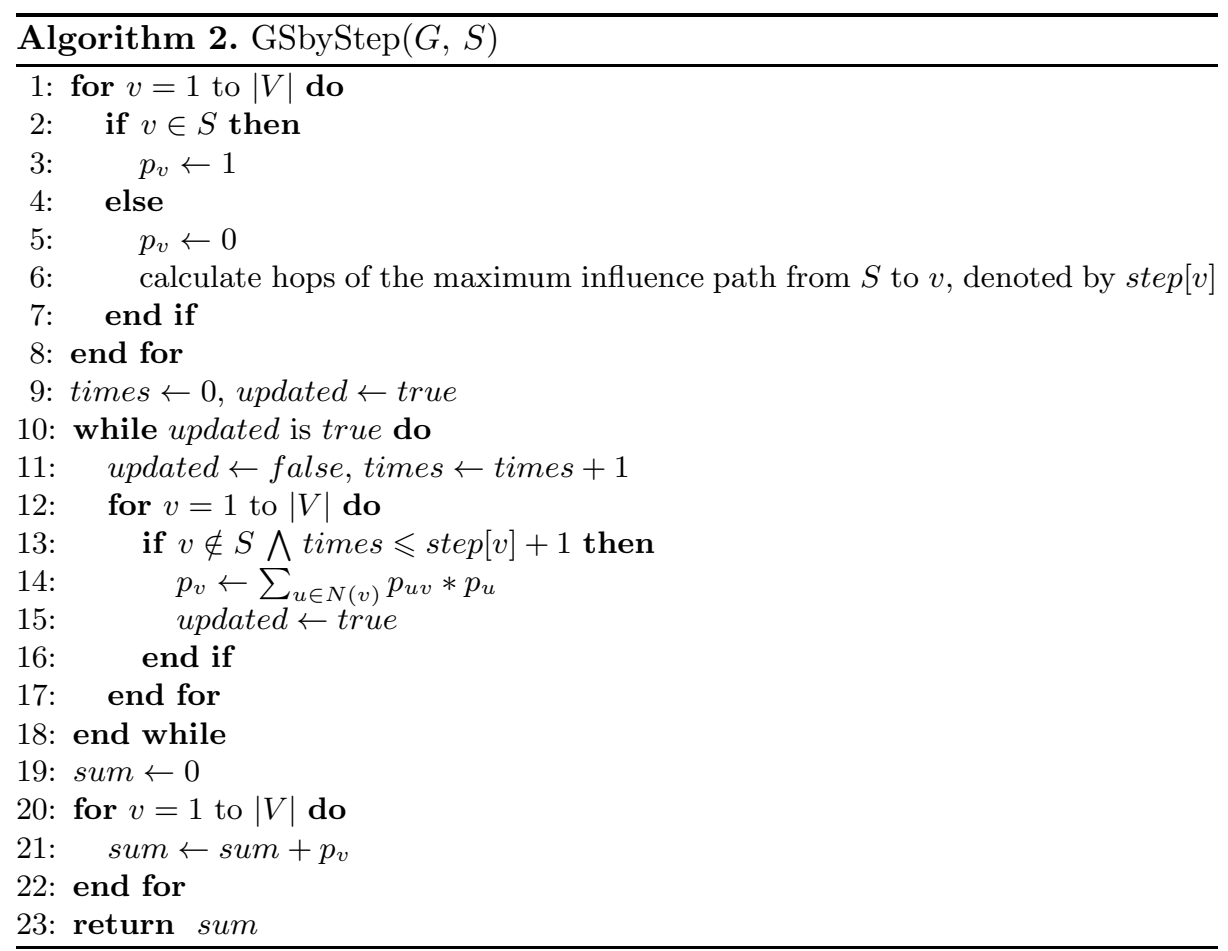


round of iterations increases, $p_{5}$ will become larger and the structural defect caused by node 5 for $p_{4}$ will be more and more serious.

To that end, as a tradeoff, we choose $\operatorname{step}[v]+1$ as $v$ 's iteration threshold $\beta(v)$. Choosing such value is because in the $(\operatorname{step}[v]+1)$-th iteration there are few miss counted influence probabilities, and meanwhile, the bad impact from structural defect is limited. Along this line, by incorporating MIP heuristic into Algorithm 1 and SteadyStateSpread, we propose GSbyStep algorithm(as illustrated by Algorithm 2) and SSSbyStep algorithm 3 for better approximations. Worth noting that our solution also works for some other iterative algorithms, where the structural defect problem exists.

\section{$5 \quad$ Experimental Evaluation}

\subsection{Experimental Setup}

We evaluate our algorithms by experiments on four real-world datasets (two directed networks and two undirected networks) that we downloaded from SNAP 4 . Detailed information of these four data sets can be seen in Table 2.

Table 2. Description of Data Sets

\begin{tabular}{|c|c|c|c|c|}
\hline Name & Description & Type & Nodes & Edges \\
\hline wiki-Vote & Wikipedia who-votes-on-whom network & Directed & 7,115 & 103,689 \\
\hline p2p-Gnutella04 & Gnutella peer to peer network from August 4 2002 & Directed & 10,876 & 39,994 \\
\hline email-Enron & Email communication network from Enron & Undirected & 36,692 & 367,662 \\
\hline ca-AstroPh & Collaboration network of Arxiv Astro Physics & Undirected & 18,772 & 396,160 \\
\hline
\end{tabular}

Unlike Kimura and Saito using uniform IC model [13] where propagation probabilities are all the same, for simulating the real-world influence propagation process more accutately, we evaluate our algorithms following more general cases of IC model. Specifically, we set propagation probabilities in a WC model [12] like way. As stated in the related work section, the propagation probability of an edge $(u, v)$ under $\mathrm{WC}$ model is weight $(u, v) /$ indegree $(v)$. Thus, this model assumes that the total influence probabilities of a node's in-neighbors are 1. As we have discussed in Section 3.1, this assumption is too idealistic for real scenarios. In order to make the influence propagation process more realistic, and following Definition 1, we slightly change WC model by setting $\sum_{u \in N(v)} p_{u v}=\alpha$, thus $p_{u v}=\alpha *$ weight $(u, v) /$ indegree $(v)$. In this way, we can not only cover more general cases of IC model (by slightly changing weights of the edges) but also capture the characteristics (i.e., small and different values) of the real-world influence propagation probabilities. Thus, in the following, we call such modified model as General IC Model, or IC model for short.

\footnotetext{
${ }^{3}$ Modifying the 14-th line of GSbyStep algorithm by replacing $\sum_{u \in N(v)} p_{u v} * p_{u}$ with $1-\prod_{u \in N(v)}\left(1-p_{u v} * p_{u}\right)$, we get the SSSbyStep algorithm.

4 http://snap.stanford.edu
} 
For each network, we conduct three groups of experiment by setting $\alpha$ as 0.5 for all nodes, setting $\alpha$ as 0.75 for all nodes, and randomly generating $\alpha$ with a uniform distribution on $[0.1,0.9]$ for each node, respectively. For evaluation, the average result of 20,000 times Monte Carlo simulation of IC model (GICM) is regarded as the real influence spread. In the following, we compare our algorithms (SSSbyStep,GSbyStep,GS) with four baseline algorithms, MIP [7], SteadyStateSpread(SSS) [1], SP1M [13] and an insufficient times Monte Carlo simulation of IC model, i.e., 200 times of Monte Carlo simulation(GICM200).

\subsection{Ranking Problem}

First we consider the problem of extracting influential seeds by ranking candidate nodes. Specifically, we try to find the rank list of $100(|S|=100)$ influential seeds, i.e., the top-100 nodes with higher influence spread than others.

We use the result of 20,000 times Monte Carlo simulation as the ground truth, and the ranking according to such simulation is regarded as the true nodes rank. For evaluating rankings obtained by different algorithms, we compare similarities between those rankings with the true ranking. We employ the same measurement which is used in [13], the ranking similarity $F(k) . F(k)$ quantifies the degree of similarity between two ranking methods at rank $k$, and it is defined as follows: Let $L(k)$ and $L^{\prime}(k)$ be the respective sets of top- $k$ nodes for the two ranking methods that we compare. Then, $F(k)=\left|L(k) \bigcap L^{\prime}(k)\right| / k$.

Fig.2-Fig.5 show the experimental results . We can see that rankings obtained by our proposed algorithms and SteadyStateSpread(SSS) are very similar
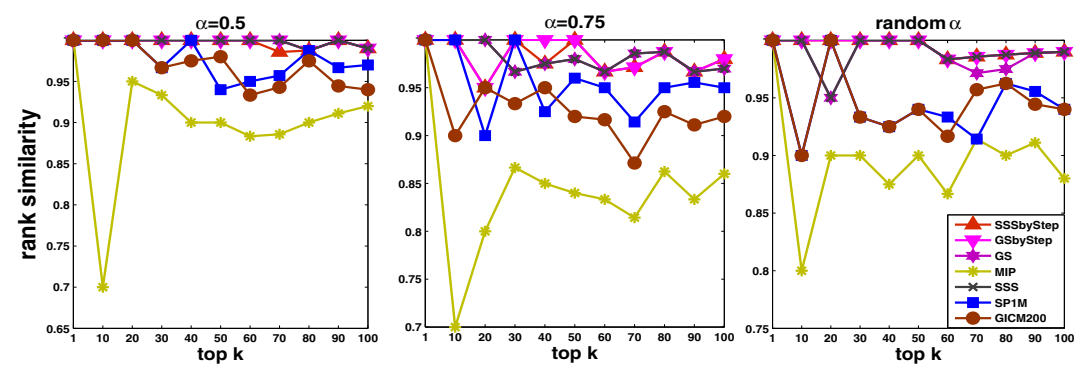

Fig. 2. Rank Similarity Comparison on wiki-Vote Network Data
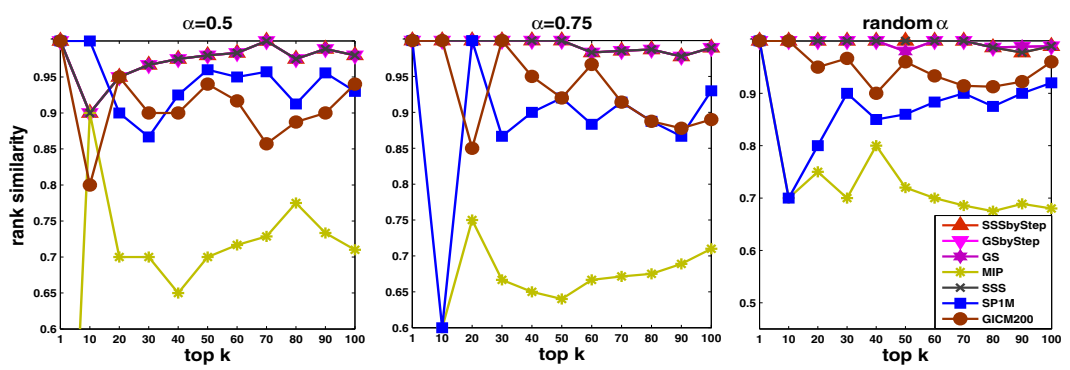

Fig. 3. Rank Similarity Comparison on p2p-Gnutella04 Network Data 

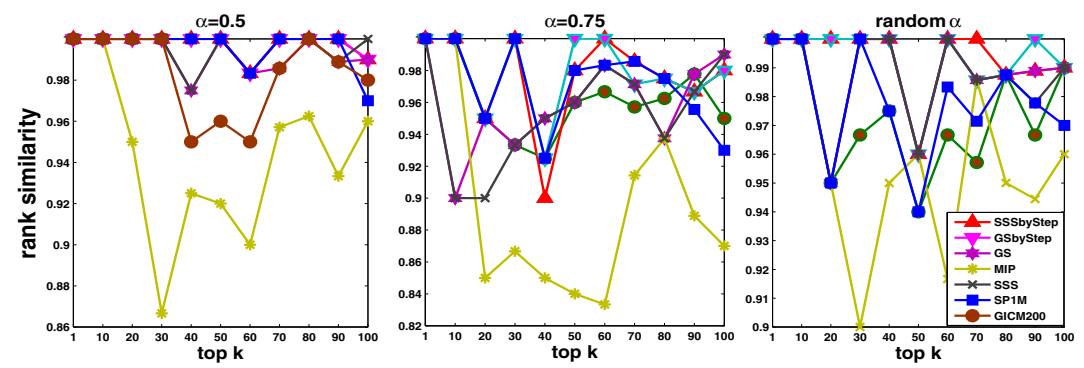

Fig. 4. Rank Similarity Comparison on email-Enron Network Data
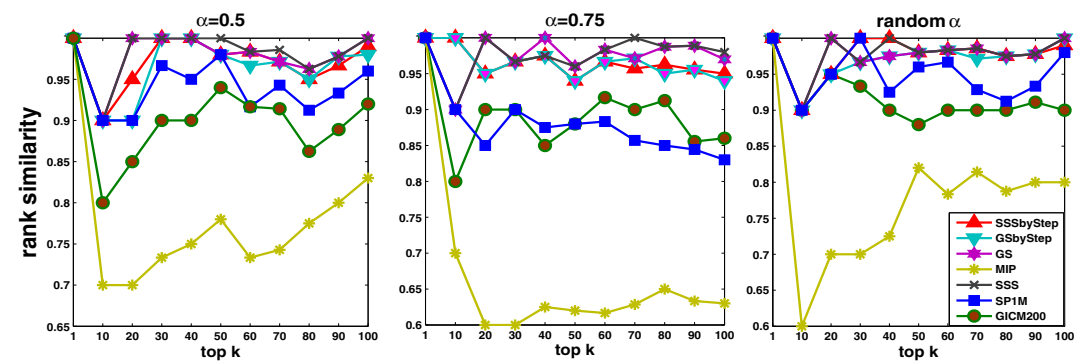

Fig. 5. Rank Similarity Comparison on ca-AstroPh Network Data

to the true ranking while others are not. This is because both our algorithms and SteadyStateSpread(SSS) are approximations for the real influence spread as stated in Section 3.

Another observation is that in most cases all algorithms can choose the most influential seed(top-1 node) accurately, even algorithms like MIP which is not very effective on ranking similarity metric. This may explain why these algorithms work well for the influence maximization problem [7][12][13]. Since under the greedy framework [12] for solving this problem, an algorithm only cares about finding the node with the maximum increasing influence spread in each round, and this node will be added into seed set.

\subsection{Estimation of Influence Spread}

We then evaluate each algorithm by their performances on estimating the influence spread for a given seed set. For each dataset, we first calculate influence spreads of the top-1000 nodes with highest out-degrees 5 , i.e., experiment with setting the size of seed set equals to 1 . Then we randomly generate 100 different seed sets with the size to be 10, 20, 30, 40, 50, respectively. At last, the effectiveness of each algorithm is measured by error rate of the influence spread returned by this algorithm. Here, we define $\sigma(S)$ as the true influence spread

${ }^{5}$ Since people often care about top influential nodes, and nodes with higher out-degree are usually more influential. 
Table 3. Average Influence Spread $(\sigma(S))$ under $\alpha=0.5$ Setting

\begin{tabular}{|c|c|c|c|c|c|c|}
\hline size of seed set & 1 & 10 & 20 & 30 & 40 & 50 \\
\hline wataset & 4.58 & 45.22 & 87.83 & 133.25 & 178.60 & 222.61 \\
p2p-Gnutella04 & 3.76 & 37.34 & 74.15 & 112.60 & 148.97 & 185.13 \\
email-Enron & 14.21 & 151.34 & 269.06 & 428.79 & 559.47 & 733.68 \\
ca-AstroPh & 4.41 & 44.06 & 86.04 & 129.91 & 171.13 & 215.14 \\
\hline
\end{tabular}

Table 4. Average Influence Spread $(\sigma(S))$ under $\alpha=0.75$ Setting

\begin{tabular}{|c|c|c|c|c|c|c|}
\hline size of seed set & 1 & 10 & 20 & 30 & 40 & 50 \\
\hline dataset & 8.29 & 80.76 & 156.41 & 234.35 & 310.25 & 384.17 \\
p2p-Gni-Vote & 8.91 & 89.58 & 169.30 & 260.26 & 343.52 & 419.03 \\
email-Enron & 31.04 & 318.28 & 579.39 & 895.69 & $1,160.61$ & $1,495.89$ \\
ca-AstroPh & 10.65 & 104.97 & 201.66 & 301.34 & 392.89 & 485.68 \\
\hline
\end{tabular}

Table 5. Average Influence Spread $(\sigma(S))$ under random $\alpha$ Setting

\begin{tabular}{|c|c|c|c|c|c|c|}
\hline size of seed set & 1 & 10 & 20 & 30 & 40 & 50 \\
\hline dataset & 4.71 & 46.34 & 90.73 & 136.85 & 183.16 & 227.99 \\
p2p-Giki-Vote & 3.81 & 37.74 & 75.29 & 113.77 & 151.07 & 188.41 \\
email-Enron & 14.02 & 150.40 & 264.93 & 424.46 & 552.89 & 724.63 \\
ca-AstroPh & 4.39 & 44.09 & 85.69 & 129.41 & 170.09 & 212.86 \\
\hline
\end{tabular}

computed by 20,000 times Monte Carlo simulation, and $\sigma^{\prime}(S)$ as the influence spread calculated by an approximate algorithm. Then the error rate of $\sigma^{\prime}(S)$ can be measured by $\left|\sigma(S)-\sigma^{\prime}(S)\right| / \sigma(S)$.

Ground Truth. The average values of $\sigma(S)$ under different $\alpha$ settings are illustrated in Table 3 - Table 5. where a much larger group of nodes are finally influenced compared to the size of each seed set. Based on these tables, we then present a verification for the assumption that $p\{\tilde{v}(t-1)\}$ is very close to 1 for each $v$ and $t$. For simplicity, we take $\alpha=0.75$ and the email-Enron network (with the largest number of influenced nodes) as an example. From Table 4 we can see that when $|S|=50$, the average $p_{v}$ for each node in email-Enron network is $1,495.89 / 36,692=0.04$, thus $1-p_{v}=0.96$. Since $1-p_{v}<p\{\tilde{v}(t-1)\}$, it means $p\{\tilde{v}(t-1)\}>0.96$. Similarly, when $|S|=1$, we can get $p\{\tilde{v}(t-1)\}>0.999$. From these lower bounds we can summarize that $p\{\tilde{v}(t-1)\}$ is very close to 1 .

Effectiveness. Correspondingly, Fig.6-Fig.9 show the average error rates of different algorithms when the size of each seed set varies from 1 to 50. From Fig.6 and Fig.7 we can see that our two linear algorithms GSbyStep and GS are the most accurate algorithms for the two directed networks. Error rates of these two algorithms are lower than $1 \%$ in most cases. While from Fig.8 and Fig.9, we find that for the two undirected networks, SSSbyStep has the lowest error rate in most cases(with error rate lower than $3 \%$ ). Worth noting that, for the two undirected networks, when $\alpha=0.75$, accuracy has been significantly 

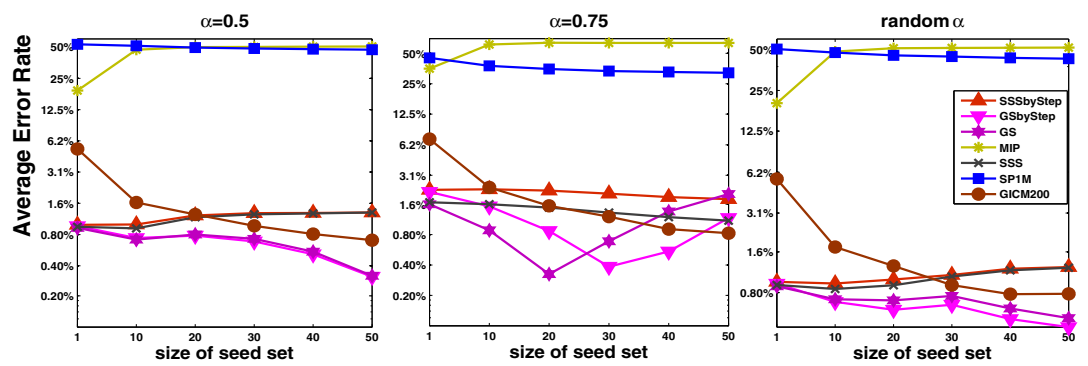

Fig. 6. Average Error Rate Comparison on wiki-Vote Network Data
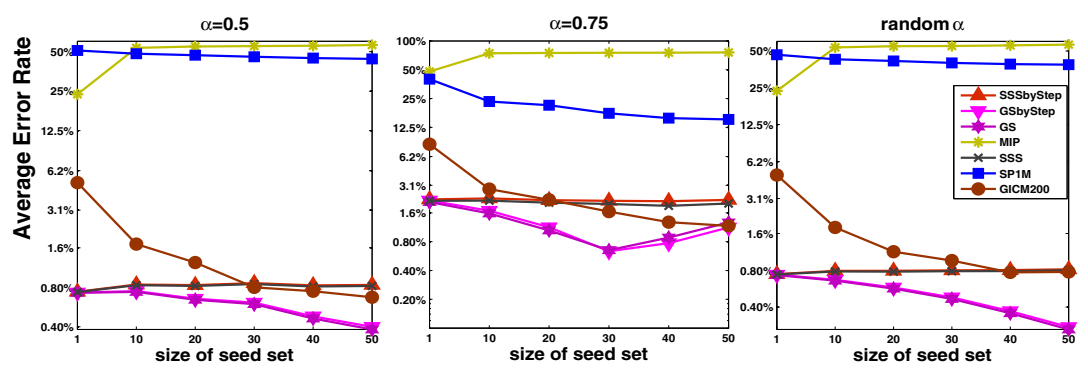

Fig. 7. Average Error Rate Comparison on p2p-Gnutella04 Network Data

improved by incorporating MIP heuristic into algorithms, especially when the size of the seed set is 1 . Specifically, average error rate of SSSbyStep algorithm is about $7 \%$ lower than SteadyStateSpread(SSS) algorithm, and average error rate of GSbyStep algorithm is about $10 \%$ lower than GS algorithm.

Another observation is that error rates of our algorithms and SteadyState$\operatorname{Spread}(S S S)$ are lower when setting $\alpha=0.5$ than setting $\alpha=0.75$. This substantiates that both our algorithms and SteadyStateSpread can better approximate the real influence spread when propagation probabilities are smaller.

Efficiency. Our algorithms are also very efficient compared to baseline algorithms. Fig.10 shows the average processing time when $\alpha=0.75$ and the size of each seed set varies from 1 to 50, where GICM denotes the algorithm of 20,000 times Monte Carlo simulation of IC model. Similar results under other $\alpha$ settings are omitted due to the space limit. We can see that tens thousand times Monte Carlo simulation ( $G I C M)$ is very time-consuming. And insufficient times Monte Carlo simulation (GICM200) is fast when the size of seed set is small, but the error rate is correspondingly very high. Moreover, another drawback of Monte Carlo simulation is that the processing time increases as seed set gets larger. In contrast, all of our algorithms are iteration-based algorithms, so the running time does not increase when seed sets become larger. 

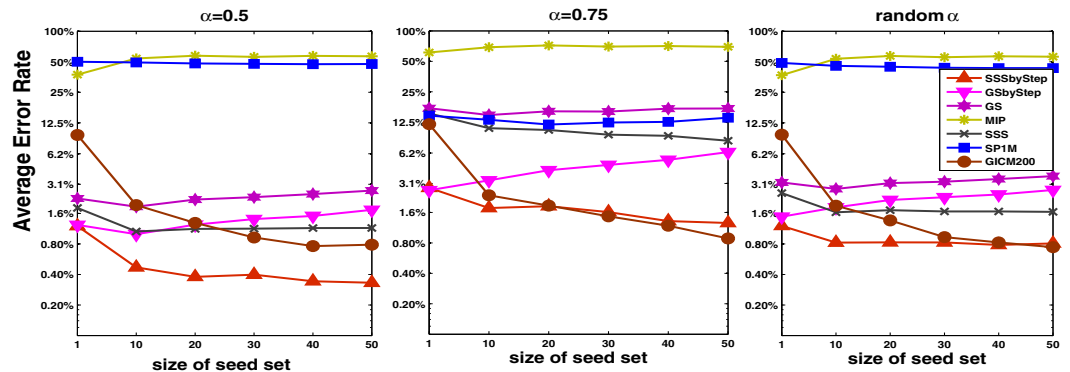

Fig. 8. Average Error Rate Comparison on email-Enron Network Data
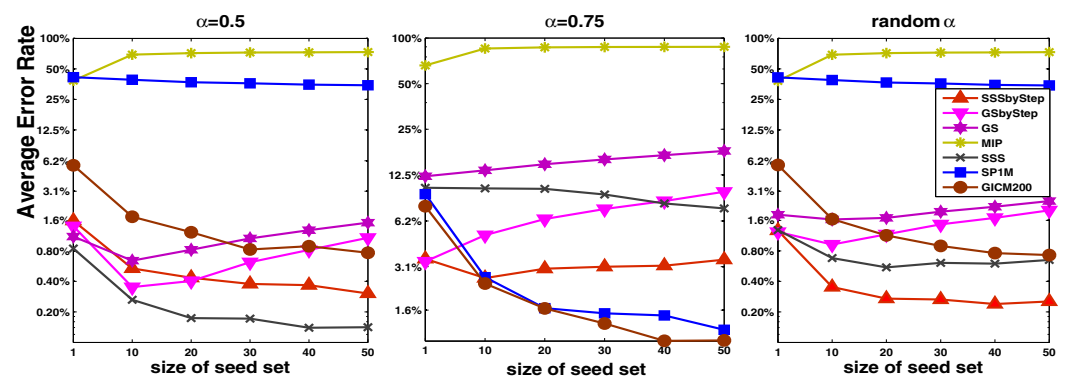

Fig. 9. Average Error Rate Comparison on ca-AstroPh Network Data

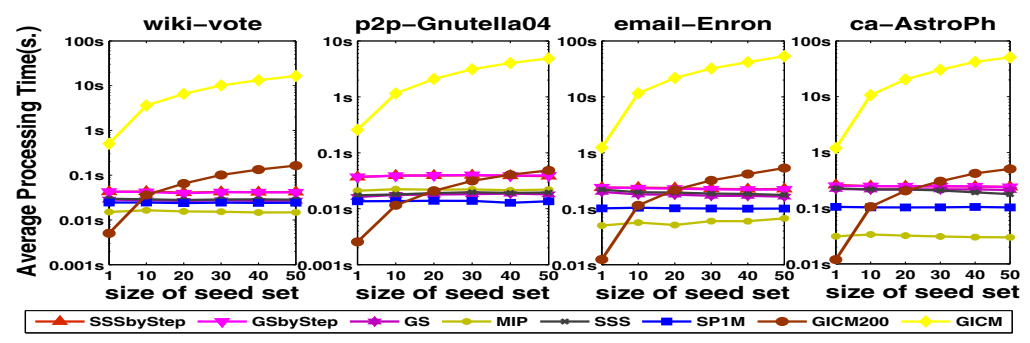

Fig. 10. Average Processing Time $(\alpha=0.75)$

\subsection{Effectiveness of Iteration Threshold}

In previous experiments, we set step $[v]+1$ as the iteration threshold for each node $v$. In this subsection, we provide an experimental proof of this value. We set $\alpha=0.75$ and use GSbyStep algorithm as an example for evaluation, and similar results can be observed for other parameter settings and algorithm SSSbyStep. Let step $[v]+r$ be the iteration threshold that we set in GSbyStep algorithm for node $v$. We did 11 groups of experiments of computing influence spreads of the 1000 nodes we picked in Section 5.3 by setting $r=0,1,2,3 \ldots, 10$, respectively. The corresponding average error rates for each $r$ are shown in Fig.11. 


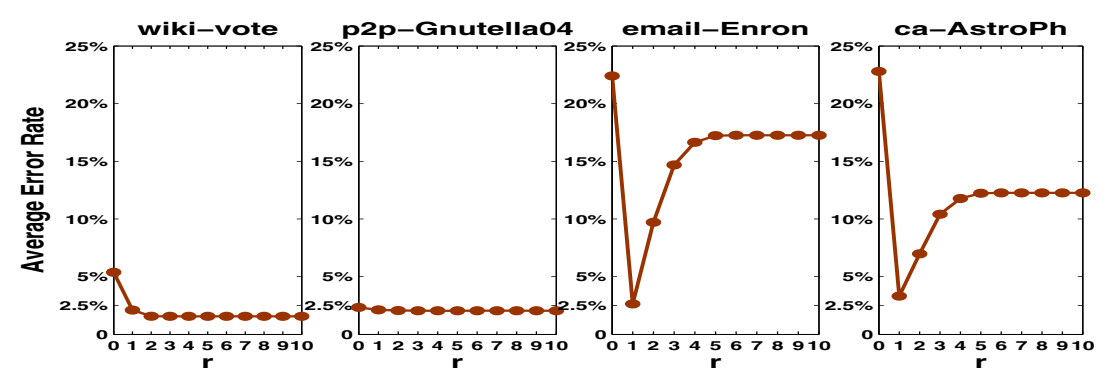

Fig. 11. Average Error Rate under Different Iteration Threshold Settings

From Fig.11 we can see that error rates under different iteration thresholds do not vary very much for the two directed networks, wiki-Vote and p2p-Gnutella04. These results are similar to that in Fig.6 and Fig.7, where the error rates of algorithms incorporating MIP heuristic(GSbyStep and SSSbyStep) are close to the algorithms not considering such heuristic $(G S$ and $S S S)$. In contrast, for the two undirected networks, email-Enron and ca-AstroPh, when setting iteration threshold to be step $[v]+1(r=1)$ we can get the smallest error rate.

In all, the above results verify that setting iteration threshold to be $\operatorname{step}[v]+1$ is reasonable for both directed and undirected networks. In this way, we can not only achieve a better approximation for the real influence spread but also has low computational cost(as shown in Fig.10).

\section{Conclusion}

In this paper, we exploited the fact that propagation probabilities in real-world social networks are quite small for developing an iterative algorithm $G S$ to quickly compute the real influence spread, under IC model. Specifically, we first explain that the influence spread can be well approximated by solving a linear system. Then, we point out the structure defect problem existing in social networks which bothers many iterative computation algorithms. Based on this discovery, we further improve both our GS algorithm and the SteadyStateSpread algorithm by incorporating the MIP heuristic. Experimental results on four real-world data sets demonstrate that $G S$ algorithm can approximate the real influence spread very well. Meanwhile, the improved algorithms(GSbyStep and SSSbyStep) can achieve better approximations and save computational cost.

Acknowledgments. The work described in this paper was supported by grants from Natural Science Foundation of China (Grant No. 61073110), The National Major Special Science \& Technology Projects (Grant No. 2011ZX04016-071), and Research Fund for the Doctoral Program of Higher Education of China (20113402110024), the Key Program of National Natural Science Foundation of China (Grant No. 60933013). 


\section{References}

1. Aggarwal, C.C., Khan, A., Yan, X.: On Flow Authority Discovery in Social Networks. In: SDM, pp. 522-533 (2011)

2. Anagnostopoulos, A., Kumar, R., Mahdian, M.: Influence and correlation in social networks. In: KDD, pp. 7-15 (2008)

3. Aral, S., Muchnik, L., Sundararajan, A.: Distinguishing influence-based contagion from homophily-driven diffusion in dynamic networks. Proceedings of the National Academy of Sciences 106(51), 21544-21549 (2009)

4. Backstrom, L., Huttenlocher, D., Kleinberg, J., Lan, X.: Group formation in large social networks: Membership, growth, and evolution. In: KDD, pp. 44-54 (2006)

5. Bakshy, E., Rosenn, I., Marlow, C., Adamic, L.A.: The Role of Social Networks in Information Diffusion. In: WWW, pp. 519-528 (2012)

6. Bus, J.C.P.: Convergence of Newton-Like Methods for Solving Systems of Nonlinear Equations. Numer. Math. 27(3), 271-281 (1977)

7. Chen, W., Wang, C., Wang, Y.: Scalable influence maximization for prevalent viral marketing in large-scale social networks. In: KDD, pp. 1029-1038 (2010)

8. Domingos, P., Richardson, M.: Mining the network value of customers. In: KDD, pp. 57-66 (2001)

9. Goldenberg, K.J., Libai, B., Muller, E.: Talk of the network: A complex systems look at the underlying process of word-of-mouth. Marketing Letters 12(3), 211-223 (2001)

10. Grosan, C., Abraham, A.: Multiple Solutions for a System of Nonlinear Equations. International Journal of Innovative Computing, Information and Control 4(9), 2161-2170 (2008)

11. Keller, E., Berry, J.: The influentials: One American in ten tells the other nine how to vote, where to eat, and what to buy. The Free Press (2003)

12. Kempe, D., Kleinberg, J.M., Tardos, É.: Maximizing the spread of influence through a social network. In: KDD, pp. 137-146 (2003)

13. Kimura, M., Saito, K.: Tractable Models for Information Diffusion in Social Networks. In: Fürnkranz, J., Scheffer, T., Spiliopoulou, M. (eds.) PKDD 2006. LNCS (LNAI), vol. 4213, pp. 259-271. Springer, Heidelberg (2006)

14. Langville, A.N., Meyer, C.D.: Deeper Inside PageRank. Internet Mathematics 1(3), 335-380 (2004)

15. Leskovec, J., Adamic, L.A., Huberman, B.A.: The dynamics of viral marketing. ACM Transactions on the Web 1(1), 5 (2007)

16. Lewis, K., Gonzalez, M., Kaufman, J.: Social selection and peer influence in an online social network. Proceedings of the National Academy of Sciences 109(1), 68-72 (2012) 